\title{
A QCD analysis of ZEUS diffractive data
}

\author{
Matthew Wing ${ }^{* \dagger}$ (On behalf of the ZEUS Collaboration) \\ Department of Physics and Astronomy, UCL, Gower Street, London WC1E 6BT, UK \\ E-mail: mw@hep.ucl.ac.uk
}

ZEUS inclusive diffractive cross-section measurements have been used in a next-to-leading-order QCD analysis to extract the diffractive parton distribution functions. Data on diffractive dijet production in deep inelastic scattering have also been used to constrain the gluon density. Predictions based on the extracted parton densities are able to describe measurements of dijet photoproduction.

XVIII International Workshop on Deep-Inelastic Scattering and Related Subjects

April 19 -23, 2010

Convitto della Calza, Firenze, Italy

\footnotetext{
*Speaker.

$\dagger$ I am grateful to Aharon Levy who stepped in to give the talk as I could not attend. My sympathies lie with the organisers of the conference who must have endured a testing time due to the disturbance caused by a volcano eruption; I am sorry that I could not come because of this.
} 


\section{Introduction}

Diffractive deep inelastic scattering (DIS) presents a very striking signature in which the proton does not break up, a large (rapidity) gap of inactivity is present in the direction of the proton and a high energy scattered electron is observed. Such processes, in which the four-momentum exchange, $Q^{2}$, of the virtual photon is sufficiently high, are amenable to predictions of perturbative QCD. The extent to which diffraction can be described by QCD is investigated here; as diffraction represents about $10 \%$ of the total DIS cross section, it is essential to understand its nature. Diffraction, according to the factorisation theorem [1], can be described as a convolution of a hard scattering process and diffractive parton density functions (DPDFs) which describe the densities of partons in the proton in a process containing a fast proton in the final state. Additionally, Higgs Bosons may be produced (at the LHC) via a diffractive process in which fast protons are detected; a deeper understanding of diffraction could therefore aid in the discovery of the Higgs Boson.

The extraction of DPDFs has been performed by several groups $[2,3,4]$ which have been able to successfully describe diffractive DIS data. Jet data have also been used [3] in fits to improve the gluon density. However, the DPDFs can not describe Tevatron data [5] on diffraction, being about an order of magnitude higher that the data. This apparent factorisation breaking can be roughly described by invoking models of secondary scatters which destroy the rapidity gap of inactivity [9]. Therefore the thrust of these proceedings [6] is to use the most precise ZEUS data on diffractive DIS and jet production in diffractive DIS [7,8] and improved theoretical assumptions to provide the best DPDFs possible to both test $\mathrm{PQCD}$ and to be used for predicting other processes.

\section{Fitting framework and procedure}

The DPDFs are parametrised as a function of the hard scale, $Q^{2}$, and the longitudinal momentum fraction, $z$, of the parton entering the hard sub-process. This assumes proton-vertex factorisation, used to model the dependence on the fraction of the momentum of the proton carried by the diffractive exchange, $x_{\mathbb{P}}$. Two contributions were assumed, called Pomeron and Reggeon, separately factorisable into a term depending only on $x_{\mathbb{P}}$ and a term depending only on $z$ and $Q^{2}$,

$$
f_{i}^{D}\left(z, x_{\mathbb{P}} ; Q^{2}\right)=f_{\mathbb{P}}\left(x_{\mathbb{P}}\right) f_{i}\left(z, Q^{2}\right)+f_{\mathbb{R}}\left(x_{\mathbb{P}}\right) f_{i}^{\mathbb{R}}\left(z, Q^{2}\right),
$$

where the Reggeon parton densities, $f_{i}^{R}\left(z, Q^{2}\right)$, were taken from a parametrisation derived from fits to pion structure-function data [10] and the Pomeron, $f_{\mathbb{P}}\left(x_{\mathbb{P}}\right)$ [7], and Reggeon, $f_{\mathbb{R}}\left(x_{\mathbb{P}}\right)$ [11], fluxes were taken from elsewhere. Next-to-leading-order (NLO) DGLAP QCD theory was fit to the data by minimising a $\chi^{2}$ using the "offset" method. The renormalisation and factorisation scales were both set to $Q$ when fitting the inclusive data and to $Q$ and the transverse jet energy, $E_{T}^{\text {jet }}$, when fitting the jet data. The general-mass variable-flavour number scheme [12] was used to account for charm and bottom of masses 1.35 and $4.3 \mathrm{GeV}$. The starting scale was $Q_{0}^{2}=1.8 \mathrm{GeV}^{2}$ with strong coupling constant, $\alpha_{s}\left(M_{Z}\right)=0.118$.

Three different fits were performed : two to the inclusive data, fits "S" and " $\mathrm{C}$ ", but with different parametrisations for the gluon density, $A_{g} z^{B_{g}}(1-z)^{C_{g}}$ or $A_{g}$, respectively; and one fitting both the inclusive and jet data, fit "SJ", with the same parametrisation as fit $\mathrm{S}$. 


\section{Fit results}

Results of the NLO QCD fit to the high-statistics inclusive diffractive DIS data at low $Q^{2}$ are shown in Fig. 1 as a function of $x_{\mathbb{P}}$ for different values of the Bjorken variable, $\beta$. Only data above $5 \mathrm{GeV}^{2}$ were used in the fit and are described well; data below $5 \mathrm{GeV}^{2}$ gave a poor fit and could not be described within this framework. Data at higher $Q^{2}$ are similarly well described. Complimentary data, where the proton was tagged event-by-event rather than statistically, determined by a lack of activity in the direction of the proton, were also well described, although they are of lower precision and hence do not constrain the fit with the same power as those of Fig. 1.

ZEUS

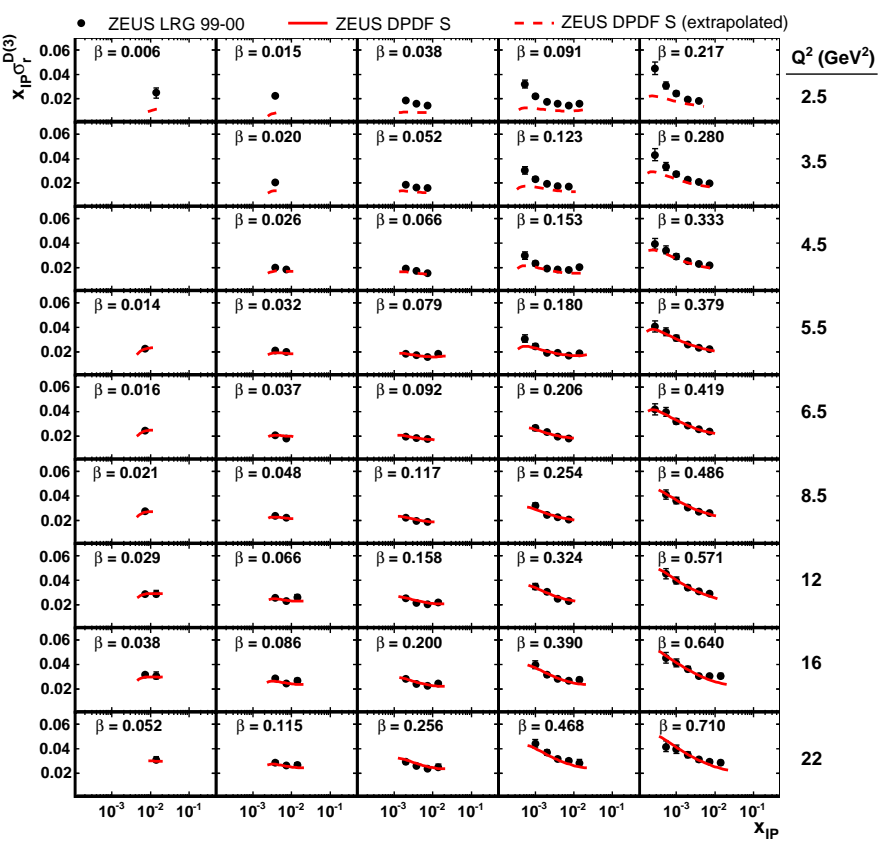

Figure 1: Comparison of NLO QCD DPDF fits to inclusive diffractive DIS data at low $Q^{2}$.

The two fits to the inclusive data only, fits $\mathrm{S}$ and $\mathrm{C}$ gave similar distributions for the quark densities with small uncertainties propagated from the experimental data. The gluon density was very different between the two, particularly at high $z$ and low $Q^{2}$. As the production of dijets in diffractive DIS is directly sensitive to the gluon content of the diffractive exchange, data on jet production were included. The fit, SJ, to the dijet data was good (see Fig. 2) with a similarly good description of the inclusive diffractive data. The gluon density from fit SJ, with comparable uncertainties to that of the quark density, is similar to that of fit $\mathrm{C}$ and fit $\mathrm{S}$ is ruled out.

\section{Comparison of fits with other data}

Figure 3 shows the fit SJ compared to the inclusive diffractive data used in the fit along with the H1 DPDF, Fit B [2] which was fit to a similar measurement from the H1 Collaboration. The $\mathrm{H} 1 \mathrm{fit}$ uses the fixed-flavour number scheme for heavy quarks, data with $Q^{2}>8.5 \mathrm{GeV}^{2}$, and were scaled to account for the different definition of the mass of the nucleon; the two fits are otherwise similar in spirit. For $\beta<0.2$, the shapes of the two fits are similar and differ by $10 \%$, consistent 


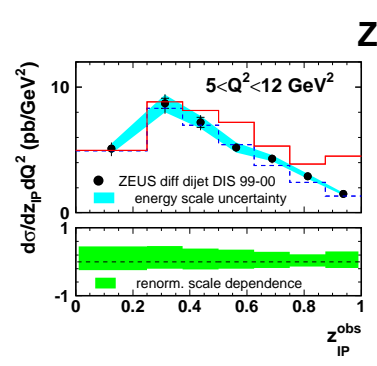

ZEUS
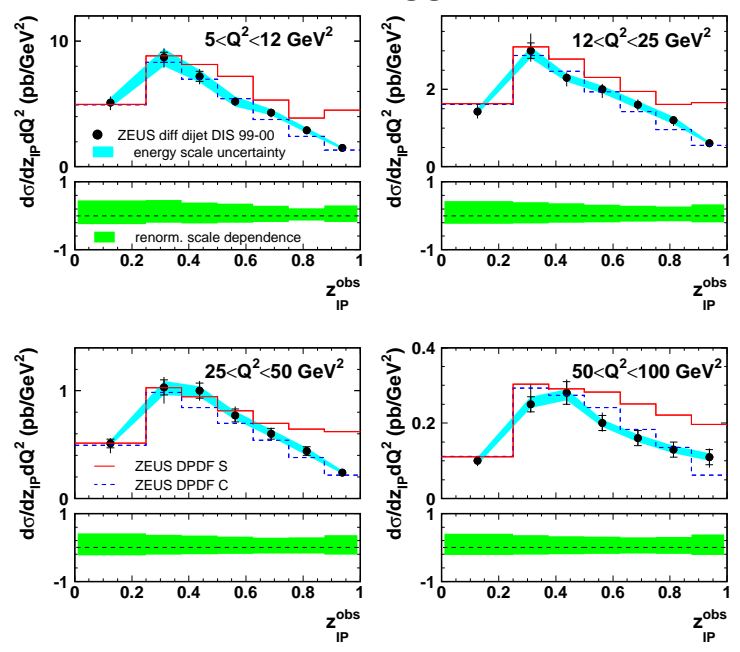

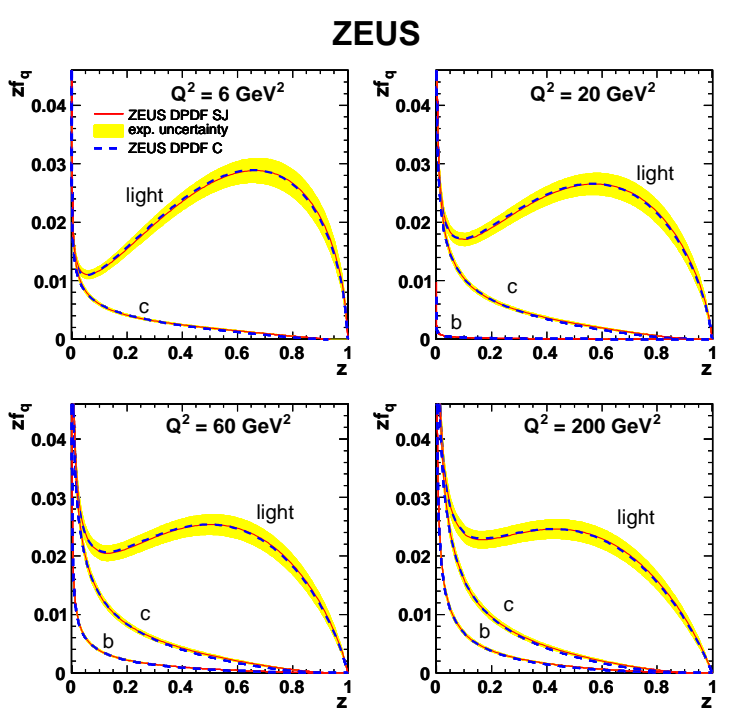

Figure 2: (Left) Comparison of DPDF fits to jet data, when not used in the fit. (Right) Resulting parton densities when including jet data into the fit along with inclusive diffractive data.

with the relative normalisation uncertainty. In regions where the fits are extrapolated, they often deviate and also at higher $\beta$ which reflects the consistency of the two data sets.

Comparison of DPDFs extracted in DIS with jet photoproduction data, in which the scattered electron escapes detection down the beam-pipe provides a powerful test of the validity of factorisation. In jet photoproduction, two processes can be considered : the direct process where all the photon's energy participates in the hard interaction and is similar to DIS; and resolved processes in which the photon develops a partonic structure and the $\gamma P$ collision is similar to a hadron-hadron collision. A measure of the fraction of photon's energy participating in the hard direction, $x_{\gamma}^{\mathrm{obs}}$, is shown in Fig. 3. The DPDF convoluted with the matrix-element calculation gives a good description of this variable with no discernible difference in the description for high- $x_{\gamma}^{\text {obs }}$ (direct) and low- $x_{\gamma}^{\text {obs }}$ (resolved) processes. The DPDFs are also able to describe charm production in DIS [6].

\section{Summary}

A QCD fit has been performed to inclusive and dijet diffractive deep inelastic scattering data. The data are well described by this fit and the quark densities (from the inclusive data) and gluon densities (from the jet data) are well constrained. The fit has to be performed for data with $Q^{2}>5 \mathrm{GeV}^{2}$ with data and fit deviating below this value; this may indicate a fundamental limit in perturbative QCD. The DPDFs can successfully predict independent measurements : charm production in deep inelastic scattering and jet photoproduction.

The resultant DPDFs are similar to those extracted previously from H1, differing by about 10\%. Clearly these improved DPDFs will not improve the description of Tevatron data in which the prediction is an order of magnitude too high. However, jet photoproduction data where a part of the cross section can be considered as a hadron-hadron interaction, and hence lead to secondary 


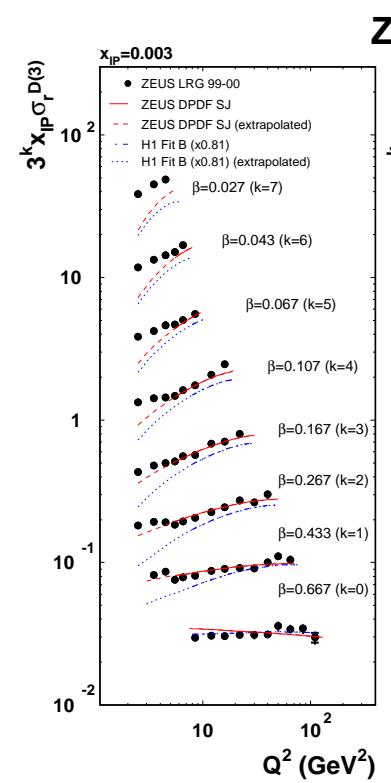

ZEUS

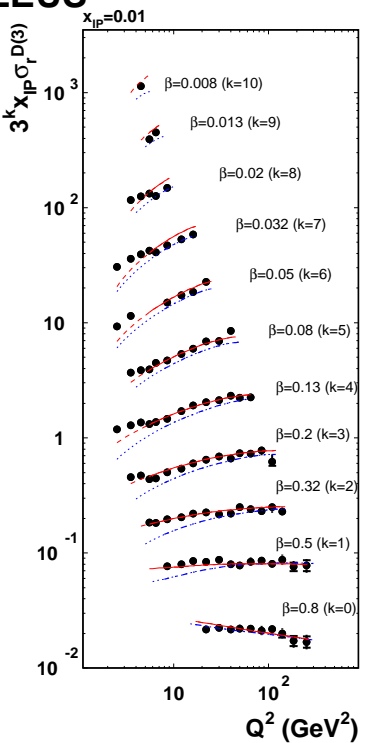

ZEUS

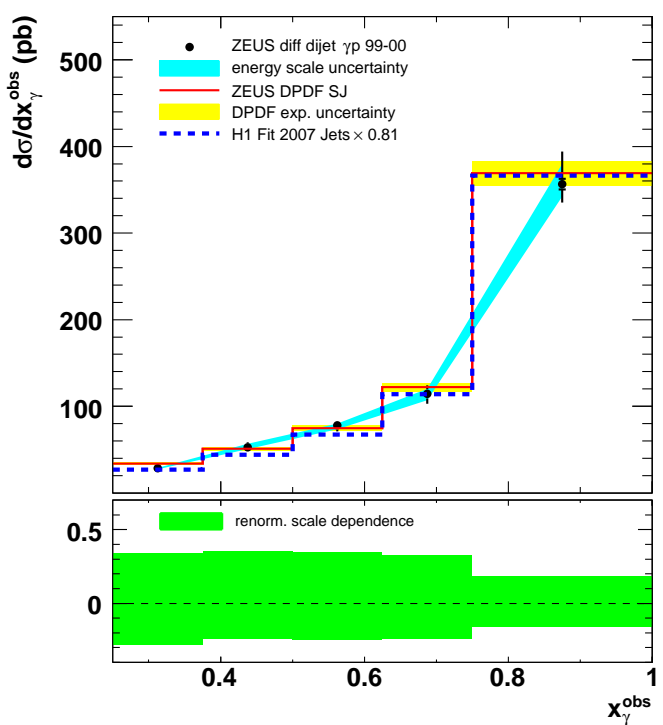

Figure 3: (Left) Comparison of ZEUS DPDF fit to the ZEUS data along with the DPDF from the H1, Fit B. (Right) Comparison of ZEUS DPDF fit with dijet photoproduction data. The fractional uncertainty due to the renormalisation scale uncertainty is also shown.

interactions, is well described by the extracted DPDFs. This does not imply a contradiction given the differences in a hadronic-photon and a hadron collision, such as the "point-like" component of the photon structure, and the different energy scales involved. Further improvements will be achieved through higher-precision measurements and understanding differences between the two collaborations and ultimately combining the data. This will put predictions of Higgs production at the LHC on a surer footing, but the reliability of these predictions is a moot point.

\section{References}

[1] J.C. Collins, Phys. Rev. D 57 (1998) 3051; Erratum, ibid. D 61 (2000) 019902; L. Trentadue and G. Veneziano, Phys. Lett. B 323 (1994) 201; A. Berera and D.E. Soper, Phys. Rev. D 53 (1996) 6162.

[2] H1 Coll., A. Aktas et al., Eur. Phys. J. C 48 (2006) 715.

[3] H1 Coll., A. Aktas et al., JHEP 0710 (2007) 042.

[4] A.D. Martin, M.G. Ryskin and G. Watt, Phys. Lett. B 644 (2007) 131; K. Golec-Biernat and A. Luszczak, Phys. Rev. D 79 (2009) 114010.

[5] CDF Coll., T. Affolder et al., Phys. Rev. Lett. 84 (2000) 5043.

[6] ZEUS Coll., S. Chekanov et al., Nucl. Phys. B 831 (2010) 1.

[7] ZEUS Coll., S. Chekanov et al., Nucl. Phys. B 816 (2009) 1;

[8] ZEUS Coll., S. Chekanov et al., Eur. Phys. J. C 52 (2007) 813.

[9] A.B. Kaidalov et al., Eur. Phys. J. C 66 (2010) 373; and references therein.

[10] M. Glück, E. Reya and A. Vogt, Z. Phys. C 53 (1992) 127.

[11] A. Donnachie and P.L. Landshoff, Phys. Lett. B 296 (1992) 22.

[12] R.S. Thorne and R.G. Roberts, Phys. Rev. D 57 (1998) 6871. 